I.

Aus der Königl. Nervenklinik zu Breslau.

\title{
Zur Diagnose der Tumoren des IV. Ventrikels und des idiopathischen Hydrocephalus nebst einer Bemerkung zur Hirnpunktion.
}

Von

K. Bonhoeffer.

(Hierzu Tafel I.)

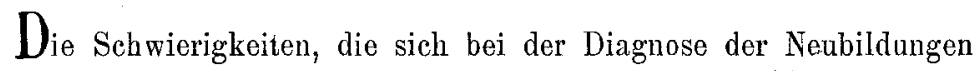
des Kleinhirns gelegentlich ergeben, liegen vor allem darin, dass innerhalb des subtentorialen Raumes jede Art der Raumbeengung zerebellare Symptome mit sich bringen kann. Jeder ausgesprochene Hydrocephalus internus, der sich auf den IV. Ventrikel erstreckt, macht, wie wir wissen, Symptome, welche oft die weitestgehende Aehulichkeit mit dem zerebellaren Tumor haben. Insbesondere zerebellare Ataxie findet sich, auch ohne dass allgemeine Benommenheit besteht, auch beim Hydrocephalus. Abduzensparesen, konjugierte Blickschwäche, Kornealreflexstörungen, nystaktische Zuckungen, Herabsetzung und Fehlen der Kniereflexe finden sich hier wie dort. Hat man die ganze Krankheitsenwicklung mit der Symptomaufeinanderfolge vor Augen, so ist man unter Umständen in der Lage, die lokalen Tumorsymptome von denen des sekundären und idiopathischen Hydrocephalus zu unterscheiden. Häufig genug ist das aber nicht der Fall und man ist lediglich auf das augenblickliche Zustandsbild angewiesen, das keine sicheren differentiellen Kriterien bietet. Diese Dinge sind durch die Arbeiten Oppenheims, Finkelnburgs u. a. hinlänglich bekannt.

Dass auch die vom IV. Ventrikel selbst ausgehenden Neubildungen dieselben Schwierigkeiten der Differentialdiagnose machen können, ist anatomisch einleuchtend; doch geht hier die allgemeine Meinung mehr dahin, dass die Diagnose dieser Neubildungen mit ziemlicher Sicherheit gestellt werden könnte (Stern).

Es sollen hier einige Beobachtungen mitgeteilt werden, die geeignet sind, die Schwierigkeit dieser Differentialdiagnose zu zeigen. Es sind 
3 Fälle von Tumoren des IV. Ventrikels, die unter sich in wesentlichen Punkten symptomatologische Uebereinstimmung zeigten, von der Lehrbuchsymptomatologie der Neubildungen des IV. Ventrikels aber abwichen. Ihnen habe ich zwei $\left.{ }^{1}\right)$ Fälle idiopathischen Hydrocephalus angereiht, die ihrerseits wieder grosse Aehnlichkeit mit den vorgenannten zeigten. Es ist in einem Teil dieser Fälle durch Hirnpunktion versucht worden, die dem Hydrocephalus entstammenden Symptome durch Liquorentleerung von den eigentlichen direkten Tumorsymptomen zu trennen. In wie geringem Masse das gelungen ist, zeigen die nachfolgenden Krankengeschichten.

1. Aug. Schm., 20 Jahre alt. Fing an Ostern 1907 „magenkrank" zu werden. Häufiges Erbrechen nach dem Essen, bitteres Aufstossen, Verschlimmerung im September desselben Jahres.

Wurde drei Monate in einem Krankenhause auf Gastroektasie behandelt. Hier trotz strenger Diät häufiges Erbrechen. Nabm um annähernd 10 Pfund an Gewicht ab.

Am 27. 3. 08 in die Klinik aufgenommen. Hier machte sie folgende Angaben: Erbrechen und Kopfschmerzen seien im April 1907 die ersten Erscheinungen gewesen. Die Kopfschmerzen seien rechts in der Stirn oft sehr heftig gewesen, so dass sie laut aufschreien musste. Das Erbrechen kam anfangs hauptsächlich nach dem Essen, später auch unabhängig davon selbst in der Nacht. Im Juni 1907 kamen Schwindelanfälle hinzu, sie musste sich festhalten, wurde halb ohnmächtig, nach wenigen Minuten war es vorüber. Solche Anfälle kamen etwa ein- bis zweimal wöchentlich.

Anfallsweise traten sehr heftige Schmerzen im Genick auf. Zuweilen bestanden Schmerzen zwischen den Schulterblättern und auch im Ieibe.

Von August $1907 \mathrm{ab}$ blieben die Menses aus. Von November $1907 \mathrm{ab}$ wurde das Sehen schlechter, zunächst nur auf dem rechten Auge. Das linke Auge wurde erst 5 Tage vor der Aufnahme schlecht. Das Gehör war gut. Etwa 8 Tage vor der Aufnahme trat ein Anfall auf, in dem es sie „wie im Schüttelfrost ergriff. Es trat ein furchtbares Sausen in den Ohren auf, als sollte der Kopf platzen, alles war steif"; sie konnte sich nicht bewegen, den Kopf nicht rühren. Das Bewusstsein war aber nicht ganz weg.

Schon seit September 1907 lag sie meist, fühlte sich schwach, wenn sie aufstand.

Das Gewicht nahm innerbalb des Jahres nach der oben erwähnten interkurrenten Zunahme um 30 Pfund $a b$.

Luetische Infektion wird in Abrede gestellt.

Befund: Der Kopf wird steif, etwas nach vornüber geneigt gehalten. Nackenmuskulatur ist angespannt, nicht druckempfindlich. Widerstand bei

1) Ein dritter Fall ist als Nachtrag bei der Korrektur geschildert. 
passiven Bewegungen, zuweilen ein zuckender Schmerz. Klopfempfindliche Punkte rechts an Scheitel- und Stirngegend und am Processus mastoideus.

Die Trigeminusaustrittsstellen I. und II. druckempfindlich.

Beiderseits Staungspapille rechts stärker als links mit Blutungen und beginnender Atrophie.

Beim Blick nach links nystagmusartige Zuckungen in den Endstellungen, rechts Abduzensparese. Leichte Parese des rechten Mundfazialis. Kornealreflex ist rechts sehr schwach, links in Ordnung. Die Zunge wird etwas zitternd und unsicher horausgestreckt, keine Deviation. Rachenreflex schwach.

Gehör ist gut. Otoskopischer Befund in Ordnung.

Geruch und Geschmack nach Angabe der Pat. in Ordnung.

Leichter 'Tremor der Extremitäten. Diffuse Schwäche der Extremitäten ohne Prädilektionstypus. Keine Ataxio in Rückenlage. Keine Veränderung der passiven Beweglichkeit. Lebhafte Patellar- und Achillesreflexe. Kein Babinski. Schwache Fusssohlen-, normale Bauchdeckenreflexe. Die Pat. geht mit vornübergebengtem Kopf, taumelt gelegentlich. Beim Stehen mit geschlossenen Aug'en Taumeln mit wechselnder Intensität. Temperatur 37,3. Keine Verlangsamung und abnorme Spannung des Pulses.

Psychisch ist die Pat. etwas verlangsamt in den Reaktionen, doch nicht eigentlich benommen; wenig Klagen.

In den folgenden Tagen anfallsweise auftretende starke Kopfschmerzen, Brechreiz, Nackenschmerz und Schwindel. Blut und Spinalflüssigkeit: negative Wassermannsche Realition. Bei der Spinalpunktion am 29. 3. starker Druck, keine Lymphozytose. $3 \mathrm{ccm}$ entleert.

30. 3. Schwierigkeit beim Wasserlassen.

31. 3. Sehr starke Kopfschmerzen. Erbrechen, Puls verlangsamt, ca. 60.

1. 4. Sehr starke Kopfschmerzen, stöhnt viel, liegt auf der linken Seite, den Kopf nach links and hinten gebeugt. Starkes Schlucken.

Parese des rechten Abduzens, rechter unterer Fazialis wie fräher. Beide Kornealreflexe schwach, links noch schwächer als rechts. Blickbewegungen nach links mangelhaft.

Unsicherer Gang, Neigung nach links abzuweichen. Singultus. Pulsbeschleunigung.

Hirnpunktion des linken Seitenventrikels. Entleerung wasserheller klarer Ventrikelflüssigkeit. Im Zentrifugat wenige Lymphozyten. Punktion des rechten Ventrikels. Entleerung von blutig gefärbter Flüssigkeit im ganzen etwa $50 \mathrm{ccm}$. Keine Tumorsubstanz.

Nach der Panktion werden die Nackenschmerzen geringer, Pat, fühlt sich auch sonst freier, weniger Kopfschmerz. Gegen Abend wieder Klagen usw. Kopfschnerzen. Abends $10 \mathrm{Ubr}, 7$ Stunden nach der Hirnpunktion, plötzlicher Exitus.

Obduktionsbefund: An den vegetativen Organen nichts Wesentliches. Schädeldach schwer, sklerosiert, geringe Diploe. Dura verdünnt, lässt Windungen durchscheinen, mässig gespannt. Sinus longitudinalis frei. Infundibulum stark vorgewölbt. Kleinhirn trichterförmig in das Foramen magnum eingepresst. 
Es entleert sich reichlich Liquor. Starker Hydrocephalus internus des III. und des Seitenventrikels. Abgesehen von der hydrocephalen Erweiterung: äusserlich keine Formveränderung an Gehirn und Kleinhirn erkennbar. Gehirngewicht 1090.

Bei Abhebung des Kleinhirns von der Medulla erscheint eine Tumormasse. Nach Durchschneidung des Wurms zeigt sich der IV. Ventrikel erweitert and ein gut wallnussgrosser, an der Oberfläche schmutzig grauer Tumor frei in den Ventrikel hineingewachsen mit höckeriger Oberfläche. Er ist mit dem Ependym von der Spitze des Calamus scriptorius bis zum Aquaeductus verwachsen. Dieser ist erweitert und durchgängig. Nach rechts bleibt der untere Kleinhirnstiel frei, nach links wächst der Tumor weiter und erstreckt sich bis zum Flocculus. Das Kerngebiet der Medulla ist vom Tumor nicht durchwachsen.

Mikrostopisch: Zellreiches Gliom (Taf. I, Fig. I).

2. C. Mor., 20jähriger Streckenarbeiter. Vater war Trinker. Selbst gut entwickelt. In der Schule gut gelernt. Nach der Schule Fabrikarbeiter. Seit einem Jahre Streckenarbeiter. Seit Februar: 1910 mit Kopfschmerzen erkrankt. Die Schmerzen sollen in den Schläfen am stärksten gewesen sein. Die Schmerzen waren zunächst morgens am heftigsten, liessen mittags nach, ganz verschwanden sie nie. Mit den Schmerzen im Kopf traten Nackenschmerzen auf, Pat. musste den Nacken steif halten. Die Nackenschmerzen wechselten an Intensität. In der ersten Zeit auch viel Erbrechen insbesondere morgens, mitunter mehrere Stunden hintereinander. Auch Gähnen und Aufstossen waren häufig. Das Erbrechen wurde besser, die Kopfschmerzen sohlimmer. Mit den Nackenschmerzen zusammen spürte Pat. auch solche in beiden Schultern, die rom Nacken ausstrahlten. Die Scbmerzen in den Schultern nahmen etwa eine Woche vor der Aufnahme in die Klinik zu.

Pat. wurde zunehmend schwächer und taumelte beim Gehen.

Aufnahme 22.4. 10. Schwächlicher Mensch, rachitischer Thorax. Innere Organe ohne pathologischen Befund. Urin frei von Zucker und Eiweiss. Puls 78 pro Minute. Temperatur 36,4. Genitalien von normaler Entwicklung. Heftige Klagen über Schläfonkopfschmerz und Nackenschmerz und gelegentlichen Schwindel. Gibt über seine Beschwerden gut Auskunft, ist nicht benommen.

Pat. hält den Kopf nach hinten und links geneigt, bewegt ihn möglichst wenig. Die passive Beweglichkeit des Kopfes ist nach allen Seiten beschränkt.

Die Austrittsstellen der Occipitales sind stark druckempfindlich. Kopf nicht klopfempfindlich. Trigeminusaustrittsstellen druckempfindlich.

Das linke Schulterblatt steht etwas höher als das rechte. Es bestebt kein Unterschied in der Kraftleistung zwischen links und rechts im Cucullaris. Anch elektrisch keine Differenz. Der linke Sternokleidomastoideus ist schwächer als der rechte, auch hier kein Unterschied in der elektrischen Erregbarkeit, die beiderseits gut ist.

Hirnnerven: Der Geruch ist in Ordnung, beginnende Optikusatrophie links, Stauungspapille im Rückgang; rechts neuritische Atrophie. Visus in Ordnung (Dr. Lenz). Links besteht ein leichter Exophthalmus. 
Die Augenbewegungen sind rechts und links beschränkt, nach rechts mehr als nach links.

Der Kornealreflex ist links herabgesetzt. Zeitweise werden Stiche links weniger deutlich empfunden als rechts.

Der Fazialis zeigt wechselndes Verhalten, im ganzen ist der linke Mundfazialis etwas schlechter innerviert als der rechte. Gelegentlich tritt dasselbe Verhalten am Stirnast hervor.

Untersuchung von Ohr und Kehlkopf ergibt keinen abnormen Befund.

Der Geschmack ist nicht gestört.

Die Zungenbeweglichkeit ist in Ordnung.

Die Sehnen- und Hautreflexe zeigen zu Anfang keinen abnormen Befund. Es besteht kein Babinskischer Reflex, kein Oppenheimscher Reflex usw. Kein Pyramidenprädilektionstypus. Im Gang fällt zu Anfang Neigung nach rechts zu taumeln auf, später wird beim Taumeln keine Seite besonders bevorzugt. Beim Stehen mit geschlossenen Augen tritt Schwanken auf. In Rückenlage besteht etwas Ataxie der Beine und Hypotonie. Keine Lageempfindungsstörung. Lumbalpunktat ergibt mässige Vermehrung der Lymphozyten, starke Eiweissvermehrung nach Nissl, Nonne, Opaleszenz.

3. 5. 10. Früh plötzlicher Benommenheitszustand, zyanotisch. Spricht verwaschen. Puls frequent, gespannt, Speichelfluss.

Nachmittags komatöser Zustand. Schwere Zyanose, röchelnde Atmung, Trachoalrasseln. Puls wie am vormittag. Auf Kampher Besserung. Nachmittags Wiederholung des Zustandes.

Abends Hirnpunktion bei der Benommenheit ohne Lokalanästhesie möglich. Punktion beider Ventrikel ohne Erfolg. Kleinbirnpunktion links. Untersuchung des entnommenen Gewebes ergibt nichts Pathologisches.

In der Nacht schnelle Besserung des Zustandes.

4. 5. Befund wie bei der Aufnahme. Doch sind die Patellarreflexe jetzt different und links besteht Babinskischer Reflex.

Abends wieder plötzliches Einsetzen desselben Znstandes tiefer Benommenheit. Starke Zyanose, Trachealrasseln, Speichelfluss, beschleunigter und stark gespannter Puls. Exitus nach 3 Stunden.

Obduktionsbefund: An den vegetativen Organen kein wesentlicher Befund. Sehr dünnes Schädeldach, an vielen Stellen durchscheinend. Dura mit dem Schädeldach nach vorn stellenweise verwachsen, gespannt. Im Sinus Iongitudinalis wenig flüssiges Blut. Gehirn abgeplattet, Pia zart, prall und trocken. Fluktuation des Ventrikels zu fühlen. Infundibulum an der Basis mässig vorgedrängt. Gehirngewicht mit Liquor $1480 \mathrm{~g}$. Kleinhirn ist in das Foramen magnum eingepresst.

Unter der verdünnten Vierhügelplatte sieht man einen blaugrauen Tumor durchscheinen.

Nach Durchtrennung des Wurmes zeigt sich, dass der IV. Ventrikel von einem Tumor ausgefüllt ist. Der unterste Teil des Calamus scriptorius ist frei. Hier liegt auch etwas Tela choroidea frei, sie geht in den Tumor über. Der Tumor ist mit dem Ependym des IV. Ventrikels verwachsen, geht aber nicht 
tiefer in die Substanz der Medulla oblongata und des Brückengrau über. Die Geschwulst hat den IV. Ventrikel vor allem nach vorn und oben gedehnt, hat den Aquädulkt erweitert und hebt das Dach des IV. Ventrikels und die Vierhügelplatte empor, etwas in sie hineinwachsend, aber sie nirgends durchbrechend. Die ganze Vierhügelplatte ist in die Länge gezogen, aber noch deutlich in den Konturen der vorderen und hinteren $Z_{w}$ eihügel erkennbar. Die Geschwulst schliesst vorn rundlich gewölbt etwas hinter der hinteren Kommissur ab. Nach hinten oben geht die Geschwulst etwas in den Unterwurm des Kleinbirns über. Histologisch kennzeichnet sich der Tumor als Gliom mit umfangreichen Blutungen, als deren Folge wohl die an einzelnen Stellen auftretenden Nekrosen der Neubildung aufzufassen sind (Prof. Winkler). Der Aquädukt ist verschlossen und im IV. Ventrikel ist nur das untere Ende, in dem etwas Tela liegt, vom Tumor freigelassen (Taf. I, Fig. II).

Das ganze Zervikalmark ist durch eine enorme Erweiterung des Zentralkanals auf mindestens das 3 fache Volum ausgedehnt. Die gesamte Rückenmarkssubstanz bildet nur einen schmalen Ring.

Die Degeneration in den Rückenmarkssträngen ist weder mit den Markscheiden-, noch mit der Marchimethode erheblich.

3. D. A., 11 Jahre. 2. 6.10 in die Klinik aufgenommen.

Von Mitte Januar desselben Jahres war aufgefallen, dass Pat. fast jeden Morgen nach dem Frühstïck erbrach. Auch in der Schule kam dies gelegentlich vor. Mitte Februar fiel er in der Schule durch Zerfahrenheit auf, während er sonst einer der besten Schüler gewesen war. Er hörte oft mitten im Diktat zu schreiben auf, schien "geistesabwesend".

Es bestanden ständig Klagen über Kopfschmerzen, er fasste sich mit beiden Händen oft an den Kopf. Es fiel auf, dass sein Gesicht gedunsen war.

Der Gang wurde von Ende Februar schwankend und taumelnd. Er konnte nicht stehen, ohne von vorne nach hinten zu fallen. Beim Gehen Neigung nach links zu fallen.

In letzter Zeit vor der Aufnahme musste Pat. zu Bett liegen.

Von Anfang März an ging er nicht mehr zur Schule, fand den Weg nicht nach Hause infolge von vorübergehenden Absenzen.

Bei der Aufnahme in die Klinik am 2. Juni 1910 zeigte Pat. kein starkes Krankheitsgefühl, er füble sich jetzt ganz wohl. Nur morgens beim Aufstehen habe er immer Kopfschmerzen. Die Schmerzen sässen über der Stirn, seien stechend. Gegen Mittag liessen sie nach. Morgens müsse er immer brechen. Manchmal werde es ihm schwindlig, so dass er umfalle. Beim Stehen sei er sehr unsicher, es werde ihm schwindlig. Das Wasserlassen gehe nur langsam.

Status: Guter Ernährungszustand, etwas gedunsenes, nicht ödematöses Gesicht. Verdicktes Unterhautgewebe. Auch die Haut an den Beinen derb, fettig infiltriert.

Innere Organe in Ordnung. Urin ist frei von Eiweiss und Zucker. Der Schädelumfang beträgt $52 \mathrm{~cm}$, linke Schädelhälfte erscheint etwas grösser. 
Perkussion des Schädels ergibt deutliches Schettern. Druckempfindlichkeit der Nähte. Kopf diffus klopfempfindlich.

Der Kopf wird nach hinten etwas steif gehalten.

Die Nackenmuskeln sind etwas gespannt. Druckschmerz der Occipitalisund Quintusaustrittsstellen.

Der Gang ist breitbeinig, Pat. taumelt bald nach rechts, bald nach links. Starkes Schwanken bei geschlossenen Augen, kann nur mit gespreizten Beinen stehen.

Ataxie der Beine in Rückenlage, auch Ataxie des Rumpfes bei Erheben aus der Rückenlage. Geringe Ataxie der Fingerbewegungen.

Ischiasphänomen. Die Muskulatur ist druckempfindlich. Die Patellarund Achillessehnenreflexe sind vorhanden, letztere gesteigert. Links Babinsk ischer Reflex.

Die passive Beweglichkeit der Extremitäten ist erhöht. Es besteht doppelseitige Staungspapille. Der Visus ist gut. Die Lichtreaktion ist herabgesetzt. Konvergenzrealition in 0rdnung. Assoziierte Blickschwäche nach rechts und links.

Der Kornealreflex zeigt sich gelegentlich links schlechter als rechts. Der linke Mundfazialis ist zeitweise schlechter als der rechte, nicht konstant.

Das Gehör ist gut. Gaumensegel, Zungenbeweglichkeit ist in Ordnung.

Liquor cerebrospinalis: geringe Lymphozytose. Nonne negativ, Nissl 2 Teilstriche, serologisch negatir.

Psychisch zeigt Pat. dauernd herabgesetzte Aufmerksamkeit, doch bostehen starke Intensitätsschwankungen. Eine genaue Sensibilitätsprüfung ist wegen der Aufmerksamkeitsstörung nicht möglich. Der Kranke sclhäft viel, ist im ganzen euphorisoh trotz starker Klagen über Kopfschmerz.

Am 9.6. wird eine Ventrikelpunktion gemacht, und zwar zunächst links. Das Schädeldach ist verdünnt. Es werden $30 \mathrm{ccm}$ leicht rötlich gefärbter Flüssigkeit entleert. Die Kopfschmerzen werden für einige Tage geringer.

Der Augenhintergrund und der objektive Befund verändern sich nicht. Die Hirnnervenparesen sind sehr wechselnd, konstant bleibt die Schwäche der Blickbewegungen nach rechts und links. Die zerebellare Ataxie nimmt zu.

Am 17. 6. Störung der Blickbewegung auch nach oben. Mehrfach Anfälle von plötzlichem Erbrechen und schlechtem kleinen Puls.

Am 29. 6. zwei Anfälle von Streckkrampf mit starker Benommenheit und Deviation der Augen nach oben. Nach dem Anfall bleibt die Benommenheit.

Nachmittags Himpunktion. Der Liquor fliesst unter starkem Druck ab.

Nach der Punktion Streckkrampf in den Beinen und den Bauchmuskeln. Beiderseits Babinski, Oppenheim, Fuss- und Patellarklonus. Weite starre Pupillen. Delirante Greifbewegungen mit den Armen. Vereinzelte Zuckungen im rechten unteren Fazialis. etwas nach.

Am 30. 6. nach guter Nacht munter, keine Kopfschmerzen. Liquor tropft

In den folgenden Tagen wieder schlafsüchtig, stark gestörte Auffassung. Somatischer Status unverändert. Starke Störung der Blickbewegung nach links mehr als nach rechts. 
Dann etwa vom 5. bis 15. 7. freieres Wesen, keine Kopfschmerzen, kein Erbrechen. Auffassung erheblich besser.

In der Nacht vom 15. bis 16. 7. Anfall von Benommenheit, kleiner schlechter Puls und Streckkrämpfe in den Extremitäten.

Ventrikelpunktion. Entleerung von $40 \mathrm{ccm}$ ohne Aspiration.

Nach der Punktion und auch tags darauf die Patellar- und Achillessehnenreflexe nicht auslösbar. Vom 17. 7. abends wieder Wohlbefinden bis zum 29. 7. abgesehen von Kopfschmerzenattaken und starken Pulsschwankungen am 25.7 .

Stauungspapille beiderseits 2-3 D, rechts eine halbe Dioptrie mehr als links.

In der Folgezeit Kopfschmerzen, stark schwankender Puls, zeitweise starkes Hungergefühl, euphorisch-scherzhaftes Wesen. Anfälle von Erbrechen und Pulsverlangsamung.

Am 13. 8. Vierte Ventrikelpunktion. Abends freies Aussehen, ungeniert dreistes Wesen, guter Schlaf. Keine nachhaltige Besserung. Am 16. 8. früh Verband und Kissen mit Liquor durchnässt. Temp. 36,4. Anfall von Streckkrampf an Arm und Bein. Bewusstlosigkeit, zweimalige Wiederholung dieser Anfälle. Nachmittags fünfte Ventrikelpunktion. Starker Druck. Spontaner Ausfluss von etwa 80-100 ccm klarer Flüssigkeit. Abends Temp. 37,4.

In den folgenden Tagen wieder normale Temperaturen. Geringer Liquorausfluss im Verband. Anfälle von Streckkrämpfen vom 20.ab, am 21.8. Exitus unter Häufung der Anfälle.

Obduktionsbefund: Schädeldach mässig dünn, Diploe fast ganz geschwunden. Nähte stark injiziert, deutlich sichtbar. Dura dünn, straff gespannt; Gehirnwindungen stark abgeplattet. Gewicht vor Liquorabfluss 1500. Pia trocken. Stichkanäle realtionslos.

Hochgradiger Hydrocephalus beider Seitenventrikel and des III. Ventrikels. Das Infundibulum ist stark vorgewölbt. Starker Druck auf die Hypophyse, die platt und tief in die Sella eingedrückt ist.

Grosser Tumor des IV. Ventrikels, der ihn ausgedehnt hat und prall ausfüllt. Mit dem Dache des Ventrikels ist er nirgends verwachsen. Er zeigt derbe Konsistenz und höckerige Oberfläche. Der Tumor sitzt dem Ependym des Ventrikels fest auf. Er erstreckt sich nicht in das Grau der Medulla oblongata, dagegen geht er etwa $1 / 2$ om oberhalb des Abduzens in das Brückengrau etwas über. Im Raum des IV. Ventrikels ist der Tumor mit dem hinteren und seitlichen Teil des linken Kleinhirnrezessus verwachsen. Rechts besteht nur eine geringe Verklebung mit dem Rezessus. Ein Zapfen des Tumors wächst unten aus dem Ventrikel, den Calamus scriptorius freilassend, heraus, drängt den Unterwurm auseinander und presst sich in das Foramen magnum hinein, das dadurch völlig verlegt wird. Der basale Teil der Medulla ist rom Tumor nicht berührt.

Histologiseh kennzeichnet sich der Tumor als zellreiches Gliom mit spärlichen Fibrillen. Die Gefässwände zeigen vielfach ausgedehnte hyaline Entartung, hier und da findet sich auch Thrombenbildung (Taf. I, Fig. III). 
Die Vierhügel sind abgeplattet. Der Aquädukt ist in die Breite gezogen und erweitert.

In allen drei Fällenthatte sich die Erkrankung unter ausgesprochenen zerebralen Allgemeinsymptomen, Kopfschmerzen, Erbrechen, Gähnen, Schlucken entwickelt. Im ersteren Falle hatte das Erbrechen zunächst Veranlassung gegeben, eine Magenaffektion anzunehmen. In allen drei Fällen stand zu Anfang neben den Kopfschmerzen das Erbrechen im Vordergrund.

Bemerkenswert ist die Lokalisation der Kopfschmerzen. Die erste Kranke hatte zunächst in der Stirn die stärksten Schmerzen, später in Stirn und Scheitelgegend, auch der dritte Kranke klagte über stechende Schmerzen in der Stirn, der zweite Kranke hatte die heftigsten Schmerzen in der Schläfe. Auch in anderen Fällen von Tumoren des IV. Ventrikels, so z. B. in Völschs Fall, findet sich die Beobachtung, dass die Kopfschmerzen nicht lediglich in den Hinterkopf lokalisiert waren. Es scheint, dass gerade der Stirnkopfschmerz nicht selten ist. Mlan wird ihn anf die begleitende Hydrocephalie zu beziehen haben und ihm ebensowenig wie der Klopfempfindlichkeit an Stirn, Scheitel oder Processus mastoideus bei bestebendem Hydrocepbalus eine lokalisatorische Bedeutung beilegen. Ausser den Kopfschmerzen bestanden in den beiden ersten Beobachtungen Anfälle von Nackenschmerz und ausstrahlenden Schmerzen zwischen den Schultern. Die Intensität der letzteren im zweiten Fall erklärt sich ohne Schwierigkeit aus der exzessiven Hydromyelie des Zervikalmarks durch Druck auf die Zervikalwurzeln. Die Druckempfindlichkeit der Quintusaustrittsstellen - eine, wie mir scheint, fast regelmässige Erscheinung bei hydrocephalem Hirndruck - ist in allen drei Fällen deutlich, in Fall 2 und 3 sind auch die Occipitales druckempfindlich. Auch das in Fall 3 bestehende Ischiasphänomen dürfte auf den gesteigerten Druck im Arachnoidealraum zu beziehen sein.

Von Stern wird als charakteristisch für die Tumoren des IV. Ventrikels die Kopfstellung nach vorne angesehen. Es soll durch diese Stellung das Volum des Schädelinneren vergrössert und dadurch der Druck vermindert werden (Henneberg). Nur im ersten Fall fand sich diese Vorwärtsneignng, in den anderen bestand die entgegengesetzte Neigung des Kopfes nach hinten. Man kann diesem Symptom der Kopfstellung nach vorn eine entscheidende Bedeutung auch dann nicht beilegen, wenn sie vorhanden ist, weil sie auch bei anders Iokalisierten Hirntumoren allerdings vor allen denen der hinteren Schädelgrube beobachtet wird.

Ueber den Zeitpunkt des Auftretens der Stauungspapille lässt sich Sicheres nicht sagen. Sie war zur Zeit der Aufnahme in allen Fällen 
ausgesprochen. Im ersten Fall sind die ersten subjektiven Erscheinungen, die auf sie hinweisen, 6 Monate nach dem Beginn der Erkrankung anfgetreten, im zweiten bestand 2 Monate nach den ersten zerebralen Erkrankungssymptomen schon in Atrophie übergehende Stauungspapille. In Fall 3 war 5 Monate nach Beginn die Stauung uasgesprochen. Die Bemerkung von Bruns, dass das Fehlen von Stauungspapille für Tumoren des IV. Ventrikels charakteristisch sei, ist demnach nicht aufrecht zu halten oder jedenfalls auf die Fälle einzuschränken, die sehr frühzeitig zum Exitus kommen und nicht zum Hydrocephalus führen.

Psychische Symptome waren im ersten und zweiten Falle kaum nachzuweisen. Nur der dritte Kranke zeigte deutlich herabgesetzte Aufmerksamkeit mit starken Intensitätsschwankungen und bei ihm bestand deutliche Euphorie, eine, wie ich glaube, ansserordentlich häufige Begleiterscheinung leichter Benommenheit.

Besonders bemerkenswert sind die in allen drei Fällen nachgewiesenen anfallsartigen Exazerbationen. Sie hatten verschiedenen Charakter. Es waren Anfälle von Brechreiz, Erbrechen, Nackenschmerz, Schwindel mit schlechtem Puls, schüttelfrostartige Zufälle mit nachfolgender Steifigkeit der Glieder unter starkem Ohrensausen, tonische Krämpfe mit Benommenheit, komatöse Zufälle mit starker Zyanose und Trachealrasseln bei gespanntem Puls.

Hinsichtlich der Herdsymptome bestand eine bemerkenswerte Uebereinstimmung.

Die Symptome der Medulla oblongata, soweit sie in Taubheit, Schlinglähmung, Dysarthrie, Aphonie, Glykosurie, Paraparesen, doppelseitiger zentripetaler Ataxie bestehen, fehlten. Es liegt das wobl daran, dass alle drei Tumoren keine Tendenz zeigten, sich in die Substanz der Medulla oblongata hineinzuverbreiten. Alle drei Kranken zeigten dagegen eine ausgesprochene zerebellare Ataxie, assoziierte Blickschwäche, herabgesetzte Kornealreflexe, zwei auch leichte Paresen des unteren Fazialis, einer eine Abduzensparese.

Gegenüber den Fällen von primärem Kleinhirntumor ist vielleicht bemerkenswert, dass das Taumeln sich erst im Verlauf der Erkrankung entwickelte.

Der Befund der assoziierten Blickschwäche in allen drei Fällen ist bemerkenswert im Hinblick auf die Angabe Uhthoffs, dass sich die assoziierte Bicklïhmung bei Tumoren des IV. Ventrikels verbältnismässig seltener finde. Es wird von der Art des Wachstums der Tumoren, die in unseren Fällen eine ausgesprochene Tendenz hatten aquäduktwärts zu wachsen und von der Intensität des Druackes, den der Pons durch die Dehnung 
des Ventrikels erfährt, abbängen, ob assoziierte Blickstörungen sich entwickeln.

Dass wir in allen drei Fällen eine Herabsetzung des Kornealreflexes auf einer Seite beobachteten, ist bemerkenswert, da das Wachstum des Tumors und gleichmässige Ausdehnung des Ventrikels die Bevorzugung der einen Seite nicht erklärte. Es kommt darin die Erscheinung zum Ausdruck, die uns bei dem Vorliegen von Hirndruck in der hinteren Schädelgrube immer wieder begegnet und auf die hingewiesen werden muss, weil auch halbseitige Symptome, namentlich, wenn sie nicht ganz konstant und sehr stark ausgesprochen sind, für die Lokalisation auf einer Seite nur sehr vorsichtig verwertet werden dürfen. Wir sehen immer wieder, dass ein anscheinend gleichmässiger Druck die einzelnen Hirnterritorien, Nerven, Kerne und Reflexe in ganz verschieden starker Weise alterieren kann, ein Punkt, der für die Frage des operativen Eingehens von grösster Bedeutung ist und der mich veranlasst, bei Tumoren der Kleinhirnsubstanz, wenn gleichzeitig ausgesprochener Hydrocephalus vorliegt und die Lokalisation ob rechts oder links von einseitigen Hirnnervenparesen abhängig gemacht werden muss, stets die Freilegung des ganzen Kleinhirns zu befürworten.

Auf eine Erscheinung, die der 3. Kranke darbot, soll noch hingewiesen werden. Es fiel bei diesem Kranken der Umgebung bald im Beginn der Erkrankung auf, dass sein Gesicht dicker wurde und bei der Untersuchung war das gedunsene, nicht ödematöse Gesicht und die starke Fettinfiltration des Unterhautgewebes höchst auffällig. Aehnliche Beobachtungen sind bei Hirndruck mehrfach gemacht worden (Schuster u. a.). Goldstein hat neuerdings von lokalisatorischen, wie mir scheint zutreffenden, Gesichtspunkten aus, diese Erscheinung näber studiert und kommt zu dem Resultat, dass es sich dabei wohl um eine durch das vorgestülpte Infundibulum verursachte Einwirkung auf die Hypophyse handelt. Unser Fall gibt einen anatomischen Beleg für diese Auffassung, insofern die Hypophyse sich bei der Obduktion tatsächlich ganz platt gedrückt erwiesen hat. Es scheint demnach die Annahme gerechtfertigt, dass es sich bei der abnormen Fettentwicklung um eine hypophysäre Reiz- oder Ausfallserscheinung gehandelt hat. Wir werden bei einem der folgenden Fälle eine ähnliche Wirkung sehen.

Die Erkrankungsdauer schwankte zwischen 4 Monaten und einem Jahr. Der Exitus erfolgte in keinem der Fälle ganz plötzlich, was zu bemerken ist im Hinblick auf die Erfahrungen bei Zystizerken des IV. Ventrikels und auch bei Tumoren des IV. Ventrikels, bei denen sonst ein plötzlicher Tod sehr häufig ist.

Weun ich die wesentlichen, allen drei Fällen gemeinsamen Symptome 
zusammen überblicke, so sind es neben den zerebralen Allgemeinerscheinungen die Anfälle von tonischen Krämpfen, plötzliche Benommenheits- und Kollapszustände, die zerebellare Ataxie, die Störung der assoziierten Blickbewegung und die Kornealreflexstörung. Es ergibt sich daraus, dass die Tumoren des IV. Ventrikels sich symptomatologisch mit irgend welcher entscheidenden Sicherheit von den Tumoren des Kleinhirns nicht differenzieren lassen. All diese Symptome, auch die anfallsartigen Exazerbationen finden wir auch bei zerebellaren Geschwülsten. Auch der Verlauf gibt keine sicheren differentiellen Kriterien. Ausgesprochene Schwankungen des Befindens, wie sie für die Zystizerken des IV. Ventrikels als charakteristisch gelten, sind nur insofern hervorgetreten, als anfallsartige Steigerungen der Symptome beobachtet wurden. Vielleicht wird man das eine sagen können, wenn bei einem Symptomkomplex, der auf die hinter Schädelgrube, speziell auf das Zerebellum hinweist, sich häufigere kỏllapsartige Zufälle, plötzliche tonische Krampfzustände zeigen, dass dann eine Beteiligung des IV. Ventrikels wahrscheinlich ist, ob durch Hydrocephalus oder durch Geschwalst wird man häufig offen lassen müssen.

Ich füge wun zwei Fälle von idiopathischem Hydrocephalus an. Auch hier war die'Differentialdiagnose des Kleinhirntumors erwogen worden, im zweiten Falle hatten wir uns allerdings mit Wahrscheinlichkeit für den idiopathischen Hydrocephalus ausgesprochen und ich habe den Kranken auch mit dieser Diagnose vorgestellt ${ }^{1}$ ).

4. 21 jähriges Mädchen M. M. Erkrankte Mitte März 1908 mit KäItegefühl, Schwindel, Uebelkeit und häufigem Erbrechen. War bis dahin, abgesehen von skrophulösem Augenbindehautkatarrh, als Kind im wesentlichen gesund, galt aber stets für blutarm. Ging am letzten März 1908 ins Bezirkskrankenhaus, hatte dort oft Kopfschmerzen, zweimal traten Krämpfe mit Bewusstseinsverlust auf, sie fühlte sich sehr müde. Etwa Mitle April wurde das Sehen schlechter, dann plötzlicher Eintritt von Blindheit, der die Ueberführüng nach Breslau veranlasste.

Aufnahme am 25. 4. 08. Status: Mittelgrosses, kräftig gebautes Mädchen, mässsiges Fettpolster, Körpergewicht $55 \mathrm{~kg}$. Innere Organe ohne pathologischen Befund. Puls 64, gelegentlich einen Schlag aussetzend. Temperatur normal. Urin frei. Blutdruck (Gärtner) $80-90 \mathrm{~mm}$.

Doppelseitige Stauungspapille mit Uebergang in Atrophie, rechts fortgeschrittener als links. Völlige Amaurose. Die Pupillen sind weit, reaktionslos (Atropinwirkung). Die Augenbewegungen sind nach rechts beschränkt, Schwäche des rechten Abduzens. Linksbewegungen der Augen besser, aber auch nicht ganz gut.

1) Verbandlungen der med. Sektion der vateriänd, Gesellsçhaft. 1908. 
Kornealreflex beiderseits schwach.

Beim Sprechen bleibt der rechte Mundfazialis etwas zurück. Zunge gerade. Rachenreflex in Ordnung.

Kraft des rechten Arms geringer als links, leichte unwillkürliche Bewegungen der rechten Hand. Feinere Fingerbewegungen rechts sehr ungeschickt, Daumenopposition sehr schlecht. Linke Oberextremität gut. An den Beinen gute Kraftleistung, keine Ataxie, kein Unterschied zwischen rechts und links, passive Bewegliohkeit in Ordnung. Patellar- und Achillesreflexe in Ordnung. Beiderseits Babinski. Banchdeckenreflex beiderseits gleich.

Beim Gehen taumelig. Beim Stehen mit geschlossenen Füssen Neigung nach hinten zu fallen.

Keine Klopf- und Druckempfindlichlseit des Kopfes. Bei Kopf bewegungen Klagen über Schwindel.

In den folgenden Tagen viel Klagen über Kopfschmerzen, dabei aber euphorisch, nicht benommen. Passt gut auf, kennt die Personen der Umgebung an der Stimme, gute örtliche und zeitliche Orientierung. Der objektive Befund wechselt, insofern Babinskischer Reflex bald vorhanden ist, bald fehlt. Die Kornealreflexe sind different, rechts schlechter als links.

Am 19. 5. folgender Befund: Nicht benommen, gute Beobachtung der Umgebung. Häufig Anfälle von Kopfschmerzen, Nackenschmerz und Schwindel. Klagt über Kältegefühl und Schmerzen in der ganzen rechten Seite. Die Opposition des rechten Daumens ist geschickter als früher, auch die linke Hand jetzt etwas ungeschickt. Tasten gut. Bewegungsempfindung anscheinend gut. Prüfung durch Aufmerksamkeitsstörung erschwert. Kraft der Hände ziemlich schwach. Kraft der Beine gut, kein Prädilektionstypus. Kein Babinski. Patellar- und Achillesreflexe in Ordnung. Beim Gehen hat sie das Gefühl, als ob es ihr die Beine unter dem Körper wegziehe, es sei als ob sie den Veitstanz in den Beinen hätte. Beim Stehen hat sie ausgesprochene Neigung nach hinten zu fallen. Das Taumeln hat zugenommen. Keine Ataxie in Rückenlage. Kornealreflex ist beiderseits gleich, Fazialis in Ordnung. Störung der Blickbewegung unverändert. Kopf klopfempfindlich. Occipitalis- und Trigeminusaustrittsstellen beiderseits druckempfindlich.

Abends Anfall von Kopfschmerzen mit Steifigkeit im rechten Bein, Kältegefühl rechts. Wälzt sich dann herum, krümmt sich im Bett.

20. 5. Klagen über Schwächegefühl der rechten Hand.

1. 6. Trotz völliger Amaurose behauptet sie gelegentlich zu sehen, offenbar halluzinatorisch. Viel Kopfschmerzen, Stechen in den Augen, oft Schwindel.

Objektiv: Differenz der Kornealreflexe von wechselnder Deutlichkeit. Geschicklichleit der Fingerbewegungen kaum mehr versohieden zwischen rechts und links. Rechts Babinski, gelegentlich anch links. Patellar- und Achillesreflex lebhaft. Blutdruck (Gärtner) 70-80 mm.

Verlangt wegen der Kopfschmerzen operiert zu werden.

6. 6. Gehirnpunktion, rechtes Kleinhirn und Stirnhirn doppelseitig. In der Tiefe von $5 \mathrm{~cm}$ im linken Stirnhirn kommt Liquor heraus. Entleerung von 4--5 com. Untersuchung des entnommenen Kleinhirngewebes ergibt normale 
Struktur. Der Liquor zeigt ganz geringe Vermehrung des Lymphozytengehalts (5-6 nach Nissl), und ist nach Wassermann negativ.

7. 6. Gibt an weniger Kopfschmerzen zu haben.

Temperatur 37,7, abends 38,1 , leichtes Oedem des linken Augenlides und der Stirn links. Am 8. 6. Temperatur 37, abends 37,6, am 9. 8. Am 9. klagt sie nicht mehr über Kopfschmerzen. Temperatur 37,0 und 37,4. Dedem des linken Lides und der Stirn besteht fort. Hat seit der Aufnahme um 17 Pfund an Gewicht zugenommen.

15. 6. Abfluss von Liquor durch die Stirnpunktionsöffnung. Fühlt sich wohl, hat seit der Punktion keine Kopfschmerzen mehr.

16. 6. Kein merklicher Liquorabfluss. Nachmittags Kopfschmerz.

17. 6. Zunehmender Kopfschmerz und Uebelkeit. Klagen über Frostgefühl, Temperatur 38,5. Punktionsstelle an der Stirn infiltriert. Inzision ergibt nichts Besonderes. Abends Temperatur 39,4. Stertoröso Atmung. Nachts plötzlicher Exitus.

Obduktionsbefund: Fettreiche Leiche. An den vegetativen Organen kein bemerkenswerter Befund, ausser einer Vergrösserung und Fettinfiltration der Leber.

Schädeldach dünn, ausgeprägt. Impressiones digitatae. Dio Punktionslöcher realitionslos. Dura sehr straff gespannt. Pia trocken, keine Trübungen. Windungen stark abgeplattet. Rechter Stirnpol mit dem Knochen verklebt. Hier lockere fibröse Verdickung der Pia. Das Infundibulum in Kirschgrösse vorgewölbt, drängt das Chiasna vor sich her. Starker gleichmässiger Hydrocephalus der beiden Seitenventrikel und des III. Ventrikels. Aquädult erweitert. Durchmesser etwa $5 \mathrm{~mm}$. Am Ependym in der Nähe der Hirnpole und auch am Occipitalpol kleinste Hämorrhagien. Ependym im allgemeinen leicht und unregelmässig verdickt, keine Granulierung. Nirgends Neubildungen. Der IV. Ventrikel erweitert, frei von Neubildungen und Verwachsungen. Tela und Plexus choroideus auch mikroskopisch ohne pathologischen Befund.

Mikroskopisch frischeste Meningitis von dem Stichkanal am Stirnhirn ausgehend.

5. 8jähriger Knabe P. F. War stets gesund gewesen. Keine Kinderkrankheiten. Erkrankte Mitte November 1907 mit Uebelkeit, Schwindelgefühl. War misslaunig, wenige Tage später plötzlich vollständige Nacken-und Rüekenstarre ohne Fieber und Kopfschmerz. Wurde vom Arzt als Caput obstipum rheumaticum mit Aspirin behandelt. Auf Aspirin Besserung und wieder subjektives Wohlbefinden, ausser Bett und vergnügt. Häufige Wiederholung solcher Anfälle bis zu zehn an einem Tage während vier Wochen. Zunehmende Verschlechterung des Gehens. Am 26. 12. 07 ins Bezirkskranlienhaus eingeliefert wegen plötzlich eingetretener schlaffer Lähmung. Dort Nystagmus festgestellt, keine Kopfschmerzen und kein Fieber, die Lähmung bildete sich schnell zurück. Erblindung trat im Laufe yon 8 Tagen ein. Erbrechen und Kopfschmerz waren niemals aufgetreten.

Aufnahme am 24. 1. 08 . 
Kopf rund, etwas vergrössert. Beim Beklopfen deutliches Schettern. Keine Klopfempfindlichleit, obere Halswirbel etwas schmerzbaft, Nacken nicht steif. Druckempfindliche Quintus- und Dccipitalisaustrittsstellen. Pupillen mydriatisch, geringe, aber deutliche Licht- und Konvergenzreaktion, leichte Abduzensschwäche rechts.

Augenhintergrund: Beiderseits in Atrophie übergegangene Staungspapille, links Rest von Lichtschein, rechts nichts. Nach ophthalmologischem Urteil keine Restitution durch Trepanation zu erwarten.

Kornealreflex beiderseits vorhanden.

Patellarreflex beiderseits normal. Achillesreflex lebhaft. Andeutung von Klonus. Kein Babinski. Bauchdeckenreflex in Ordnung. Motorische Kraft gut. Geringe Unsicherheit der oberen Extremitäten. Starke Ataxie der unteren Extremitäten in Rückenlage. Stehen für einen Moment möglich, gerät bald ins Schwanken. Gang nur mit Unterstützung möglich, ataktisch und taumelnd. Sensibilität für alle Qualitäten gut, auch Lokalisation und Bewegungsempfindung gut.

Psychisch lebhaft, intelligent, vergnügt. Schmierkur und Jod.

31. 1. Keine Aenderung, keine subjektiven Klagen. Gelegentlich Babinski rechts.

10. 2. Lumbalpunktion: Druck etwas erhöht. Flüssigkeit klar. Im Zentrifugat etwa 7 Lymphozyten im Gesichtsfeld, also eine geringe Vermehrung. Kein Fibrinnetz, geringe Eiweisstermehrung.

13. 2. Beim Gehen und Stehen fällt Pat. nach vorwärts und rückwärts.

26. 3. Angina follicularis. Kopfschmerzen, mebrfach Erbrechen. Etwas benommen.

29. 3. Angina geheilt. Anfall heftigster Kopfschmerzen mit lautem Sehreien, im Hinterkopf und Nacken lokalisiert.

Zeitweise Steifigkeit der Nackenmuskulatur und opisthotonische Haltung. Puls etwa 100, nicht ganz regelmässig.

Im Anfall vorgenommene Punktion. Die Flüssigkeit spritzt unter starkem Druck hervor. $10 \mathrm{ccm}$ abgelassen. Bald Beruhigung und Schlaf.

30. 3. Ophthalmoskopisch etwas mehr Staung.

1. 4. Kopfschmerzen abends zunehmend. Kopf nach hinten in die Kissen gebohrt. Blickbewegung nach rechts behindert, nach links gut. Beiderseits Patellar- und Fussklonus und Babinski. Stöhnt und ächzt. Beschleunigte Atrnung und beschleunigter Puls. Sensorium leidlich frei. Lumbalpunltion (10 $\mathrm{ccm}$ entnommen) bringt keine Besserung.

2. 4. Viel Nackenschmerz. Benommen, beschleunigte Atmung, schwitzt. Zunehmendes Koma. Zyanose. Reflexe verschwinden. 4 Uhr Exitus.

Obduktionsbefund: Schädel rund, Nähte klaffen etwa $1 \mathrm{~cm}$ breit. Schädeldach in toto verdünnt, der Knochen entsprechend den Windungen usuriert. Die Nahtlücken sind dureh eine stark vaskularisierte, weiche weissrötliche, osteoide Substanz ausgefüllt. An den Fontanellen kein stärlieres Auseinanderstehen der Schädelknochen.

Dura dünn, zart, durchsichtig, Subduralraum leer. 
Pia trocken, zart, keine Verdickungen. Windungen stark abgeplattet, Gyri verstrichen. Beim Durchschneiden des Hypophysenstiels fliesst aus dem vorgewölbten Infundibulum eine grosse Menge Liquor ab. Hochgradiger Hydrocephalus internus. Hinterhörner reichen bis $1 \mathrm{~cm}$ vor den Hinterhauptpol. Unterhörner für zwei Finger bequem durchgängig. Seitenventrikel-Querdurchmesser 2-3 cm, Höhe 3-4 cm. Aqqü̈dukt stark erweitert, ebenso IV. Ventrikel, die Recessus dringen bis tief ins Klejnhirn. Erhebliche ampulläre Dilatation des Zentralkanals im Rückenmark, nach unten allmählich abnehmend.

Keine herdförmigen Veränderungen am Gehirn, keine Atrophien, keine Neubildungen. Keine Veränderung des Ependyms, der Plexus and der Tela choroidea.

Somatisch: Gut genährteLeiche, Hyperämie der Leber, sonst ohne Befund.

In beiden Fällen ergab der Obduktionsbefund Hydrocephalus, obne dass sich anatomisch eine Erklärung für seine Entwicklung gab.

Klinisch zeigten die Fälle in Verlauf und Symptomatologie unter sich und mit den vorigen Fällen in wesentlichen Punkten Uebereinstimmung. Die Entwicklung war in beiden Fällen anscheinend ziemlich akut ohne ersichtliche Veranlassung, im zweiten Fall mit charakteristischen opisthotonischen Anfällen ohne Bewusstseinsverlust, im ersten mit Schwindel, Kopfschmerzen and Erbrechen und vereinzelten Krampfanfällen, über deren näheren Charakter die Angaben fehlen. In beiden Fällen wird etwa 6 Wochen nach dem Krankheitsbeginn durch die plötzlich eingetretene Erblindung der ernsthafte zentrale Charakter der Erkrankung deutlich und die Ueberführung in die Klinik veranlasst. Nach dem kurz darauf erhobenen Befund der schon in Atrophie übergegangenen Stauungspapille muss ein sehr frühzeitiger Beginn der Stauungs papille angenommen werden. Darin ein differentiell diagnostisches Kriterium gegenüber den sekundären Hydrocephalien in den vorigen Fällen zu erblicken, ist, wenn man die Kasuistik des idiopathischen Hydrocephalus überblickt, kaum angängig. Bemerkenswert ist die Plötzlichkeit der Erblindung, die auch sonst bei diesen Fällen gelegentlich beobachtet wird. Es scheint, dass der Widerstand der optischen Leitung gegen den Druck des Hydrocephalus und des Infundibulums, um deren Summation es sich wohl vorwiegend handelt, sich unter Umständen plötzlich erschöpft (vergl. Henneberg). Es liegt darin ein praktisch wichtiger Punkt für die Behandlung dieser an sich beilbaren Prozesse. Man wird sich bei der Frage der Eventualität der Palliativtrepanation nicht zu sehr auf den vorhandenen Visus verlassen dürfen, weil dieser anscheinend innerhalb gewisser Grenzen von individuellen Resistenzverhältnissen abhängig ist, sondern man wird vor allem den objektiven Befund an der Papille für die Frage des operativen Eingriffs zu beobachten haben. In beiden Fällen war beim Eintritt in die Behandiung 
von der Trepanation für die Erhaltung des Sehens nichts mehr zu erwarten. Frühzeitige Trepanation ist in diesen Fällen akuter Hydrocephalusentwicklung, wenn die andere Behandlung versagt, dringend geboten.

Das Bild bei der Aufnahme wies in anscheinend unzweideutiger Weise auf die hintere Schädelgrube, speziell das Zerebellum hin. Es dominierte unter den Lokalsymptomen hochgradige statische Ataxie, im ersten Fall unter der speziellen Form der Asynergie cérébelleuse. In beiden Fällen traten hinzu ataktische Störungen der Extremitäten, im ersten Fall halbseitiger Art in den oberen Extremitäten, im zweiten ausgesprochene Ataxie der Beine in Rückenlage. Da sie in beiden Fällen ohne Störung der Tiefenempfindung einhergingen, durften auch sie auf das Zerebellum beziehungsweise auf zerebellare Bahnen bezogen werden.

Dazu kommt in beiden Fällen assoziierte Beschränkung der Blickbewegung im zweiten Fall allerdings nur. vorübergehend, leichte Abduzensparesen im zweiten Falle auch vorübergehend Nystagmus.

Wechselnde Störungen des Kornealreflexes bestanden im ersten Fall.

Wir sehen also einen mit den Kleinbirn- und unseren Ventrikeltumoren recht übereinstimmenden Symptomenkomplex. Dazu kommt, dass bei der ersten Patientin das Brunssche Symptom des IV. Ventrikels angedeutet war, insofern sie bei Kopfbewegungen ausgesprochenen Schwindel bekam. Bei den Ventrikeltumoren selbst hatten wir das Symptom vermisst. Eine entscheidende Bedeutung nach der einen oder anderen Richtung konnte ich ihm um so weniger beilegen, als ich zwei Fälle von Kleinhirntumoren beobachtet habe, in denen es in ausgesprochener Weise vorhanden war. In einem Fall handelt es sich um einen im rechten Lobus quadrangularis sitzenden Tumor, der von Anschütz operiert worden und geheilt ist, im anderen um einen im linken Kleinhirn sitzenden grossen, in eine Cyste verwandelten Tumor. Uebrigens weist Bruns neuerdings darauf hin, dass die Ventrikelnähe eines Prozesses der hinteren Schädelgrube genüge, um die Schwindel- und Nauseaerscheinungen bei Lokomotionen hervorzurufen.

Für die Entscheidung der Frage, ob idiopathischer Hydrocephalus, ob Tumor des IV. Ventrikels, ob Kleinhirntumor bestanden im zweiten Falle eine Reihe von Wahrscheinlichkeitsgründen, den ersteren anzunehmen, die weniger in den Herderscheinungen als in den Allgemeinsymptomen und im Verlaufe gelegen waren. Bezüglich der Herderscheimungen konnte vielleicht darauf hingewiesen werden, dass sie abgesehen von der ataktischen Störung, wenig ausgesprochen und flüchtiger Art waren (Nystagmus, konjugierte Blickschwäche). Im Verlauf war bemerkenswert die schnell auftretende und wieder verschwindende 
Paraplegie, eine Beobachtung, auf die anch Oppenheim beim idiopathischen Hydrncephalus hinweist. Für den genuinen Hydrocephalus konnte auch angeführt werden das Alter des Patienten, um so mehr, da Lues und Tuberkulose ausgeschlossen werden konnten, dann die interkurrente deutliche Verschlimmerung aller Symptome anschliessend an die leichte Angina, die der Patient in der Klinik durchmachte. Die Beziehungen des idiopathischen Hydrocephalus zu Infektionskrankheiten sind bekannt.

Die Beobachtung, dass Kopfschmerzen im zweiten Falle selbst während der opisthotonischen Anfälle keine Rolle spielten, ist ungewöhnlich und kann vielleicht seine Erklärung in der Nachgiebigkeit des kindlichen Schädels gegen den steigenden Druck finden, vielleicht auch, dass ein latenter Hydrocepbalus congenitus die Volumverhältnisse in einer für eine akute Dehnung günstigen Weise vorbereitet hatte. Unsere in diesem Falle mit Wahrscheinlichkeit gestellte Diagnose auf idiopathischen Hydrocephalus hat sich bestätigt. Man wird sich aber nicht verhehlen dürfen, dass es eben nur eine Wahrscheinlichkeitsdiagnose war. Der Obduktionsbefund eines Tumors würde nicht überraccht haben. Es ist darauf geachtet worden, ob die Zusammensetzung der Spinalflüssigkeit differentiell diagnostische Anhaltspunkte geben konnte. Es hat sich aber ergeben, dass das Verhalten der Ventrikelfüssigkeit sich beim sekundären Hydrocephalus nicht anders verhielt, wie beim primären. Hier wie dort fand sich in einzelnen Fällen eine leichte Lymphozytose und Eiweissvermehrung und ebenso auch in anderen Fällen normale Liquorbeschaffenheit.

Man wird sich nach all dem zurzeit darüber klar sein müssen, dass noch immer in einer ganzen Anzahl von Fällen die Differentialdiagnose zwischen Kleinbirntumor, Tumor des IV. Ventrikels und idiopathischem Hydrocephalus nicht mit Sicherheit zu stellen ist.

Im Hinblick auf das oben über den Einfluss des Hirndrucks auf die Hypophyse Gesagte sei auf die bei der schweren Erkrankung ungewöhnliche Gewichtszunahme der Kranken 4 hingewiesen. Ihr Körpergewicht nahm in etwa 9 Wochen während des Aufenthalts in der Klinik dauernd bis zum Exitus zu und zwar im ganzen um 17 Pfund. Die Analogie mit dem Fall 3 liegt anf der Hand. Anatomisch zeigte anch hier sich das Infundibulum in Kirschgrösse vorgewölbt. Bei der Obduktion ist leider auf das Verhalten der Hypophyse damals nicht besonders geachtet worden. Man wird aber berechtigt sein, auch hier eine Druckwirkung auf diese anzunehmen und die abnorme Fettbildung auf die Hypophyse zu beziehen.

Zum Schluss noch ein Wort zu den Hirnpunktionen. 
Dass Tumoren yon der Art der hier geschilderten inoperabel sind, bedarf kaum der Erwähnung ${ }^{1}$ ). Wir haben versucht, symptomatisch gegen den Hydrocephalus durch Hirnpunktion vorzugehen und dachten dabei zugleich durch Beseitigung des Hydrocephalus vielleicht ein kilareres Symptomenbild der ejgentlichen Lokalsymptome zu bekommen. Bekanntlich hat Anton neuerdings seinen Balkenstich gerade auch von dem Gesichtspunkt aus empfohlen, weil durch ihn ermöglicht werde, die eigentlichen direkten Tumorherdsymptome von den sekundären durch den Hydrocephalus bedingten zu unterscheiden. Wie weit sich durch den Balkenstich die Jokalisatorischen Verhältnisse klar gestalten lassen, kann ich aus eigener Erfahrung nicht sagen. Die Hirnpunktion hat sich uns jedenfalls für die Förderung dieser Frage weder in den vorliegenden, noch in anderen Fällen erfolgreich gezeigt.

Auch in therapeutischer Hinsicht kann man nicht sagen, dass die Erfolge ermutigend gewesen sind. Im ersten Falle wurde, als die Nackenschmerzen sehr heftig waren, als Pulsbeschleunigung und Singultus auftrat, doppelseitige Ventrikelpunktion gemacht. Der unmittelbare Erfolg war eine Linderung der Beschwerden, aber der Exitus erfolgte 7 Stunden nach der Punktion. Wenn man auch nicht mit völliger Sicherheit sagen kann, dass der Exitus vielleicht auch ohne den Eingriff eingetreten wäre, da der Erkrankungsprozess offenbar im Zunehmen und seiner Natur nach letal war, so ist doch der zeitliche Zusammenhang so, dass eine ursächliche Mitwirkung der Punktion an dem letalen Ausgang wahrscheinlich ist. Wir wissen, dass auch sonst der Zeitraum von 6-12 Stunden nach der Punktion ein kritischer ist.

Im zweiten Fall wurde die Punktion im komatösen Zustand gemacht. Ob die nachher eintretende Besserung mit der Punktion in $\mathrm{Zu}$ sammenhang steht, ist fraglich, da die Punktion keine nennenswerte Liquormenge förderte. Dass der tags darauf erfolgende Exitus in diesem Falle mit der Punktion im ursächlichen Zusammenhang steht, ist nicht wahrscheinlich, da ein akuter komatöser Zustand sich auch schon vor der Punktion ganz in derselben Weise tags zuvor eingefunden hatte.

1) Selbst Anton, der in einer nach Abschluss dieser Arbeit erschienenen Publikation (Dieses Archiv 48. Bd. S. 529) die Operationsmöglichkeit der Tumoren des IV. Ventriksels bejaht, würde in unseren Fällen, da Verwachsungen mit dem Ventrikelboden vorlagen, die Operation ablehnen. Praktisch wird, glanbe ich, vorläufig auch in anders liegenden Fällen bei der meist vorliegenden Unmöglichkeit in vivo die Ausdehnung des Tumors im Ventrikel zu übersehen und der Schwierigkeit, die Blutung in der Tiefe zu beherrschen, die Beschränkung auf die Palliativtrepanation vorzuziehen sein. 
Man könnte hier höchstens an eine durch die Punktion herabgesetzte Widerstandskraft denken.

Im 3. Falle wurde $4 \mathrm{mal}$ der Ventrikel punltiert im Laufe von 18 Tagen. Vorübergehend schien es, als ob die Punktion eine Besserung des subjektiven Befindens veranlasst hätte. Aber ein wesentlicher und nachhaltiger Einfluss auf das Ergehen und auf das Verhalten der Stauungspapille wurde nicht beobachtet. Bemerkenswert ist in diesem Falle die Entwicklung einer Liquorfistel, offenbar bedingt durch den starken Ventrikeldruck. Die Gefahren der Liquorfistel für die sekundäre Infektion sind bekannt. Im vorliegenden Falle starb der Kranke, ehe es zu einer solchen gekommen war.

Im 4. Falle wurde die Punktion lediglich zu diagnostischen Zwecken gemacht. Bei der Stimhimpunktion gelangte man in der Tiefe in den erweiterten Ventrikel. Auch hier kam es zu einer Liquorfistel, die sich infizierte und 10 Tage nach der Punktion zum Exitus führte. Die Möglichkeit, dass die Bildung der Liquorfistel schon den Beginn der Meningitis bildete, kann nicht ganz in Abrede gestellt werden, obgleich dabei bemerkenswert wäre, dass bei der Obduktion, die drei Tage nach dem Auftreten der Liquorfistel sich einstellte, makroskopisch noch nichts von Deningitis zu sehen war.

Ich kann mich nach diesen Erfolgen nicht mehr sehr für die Ventrikelpunktion zur Beseitigung des Hydrocephalus erwärmen. Es muss aber hinzugefügt werden, dass wir in anderen Fällen nicht ganz so ungünstige Resultate bei der Punktion gesehen haben. In einem Falle von sekundärem Hydrocephalus hatten wir einen für längere Zeit vorhaltenden Erfolg. Im ganzen wird aber, wie mir scheint, der Hirnpunktion als therapeutischem Mittel gegen den Hydrocephalus keine grosse Zukunft beschieden sein. Ihre Wirkung ist von zu geringer Nachhaltigkeit und offenbar meist nicht imstande, die Entlastung bis zum Rückgang der Stauungspapille zu bringen. Kausch hat bei angeborenem Hydrocephalus mit der Hirnpunktion anscheinend bessere Erfolge gehabt. In Fällen scbweren hydrocephalen Hirndrucks bei starker Verdünnung des Hirnmantels, der Dura und des Schädels ist offenbar die Gefahr der Bildung einer Liquorfistel nicht ganz gering.

Harmloser ist die Lumbalpunktion. Sie hat uns im 5. Fall einmal vorübergehend Besserung der Opisthonusanfälle gebracht, späterhin sich aber erfolglos gezeigt. Einen Dauererfolg durch Lumbalpunlation habe ich auch sonst nie bei hydrocephalem Hirndruck gesehen, im Gegensatz zu anderen Beobachtern (Quinke, Lenhartz, Riebold u. a.). Bei Prozessen, wie den drei erst geschilderten, wo das in das Foramen magnum gedrängte Kleinhirn, die Kommunikation zwischen 
Spinalsack und Ventrikel verhindert, ist von ihr ohnehin nichts zu erwarten. Man wird, um Dauerentlastung zu erzielen, in der Mehrzahl zu eingreifenderen Methoden, zur Palliativtrepanation, event. zum Balkenstich oder zur Payrschen Drainage zu greifen haben. Ich möchte nach unseren Erfahrungen vielleicht sogar soweit gehen, zu sagen, dass man am besten die Ventrikelpunktion zur Beseitigung des Hydrocephalus überhaupt nicht oder jedenfalls nicht häufiger anwendet, da vielleicht dadurch, worauf auch 0 ppenheim hinweist, die Chancen für eine spätere Operation durch Verminderung der Widerstandskraft des Gehirns vermindert werden. Ihr Anwendungsgebiet wird vorläufig am besten auf die Diagnostik unklarer Fälle beschränkt bleiben, bei denen man aus irgend welchen Gründen nicht gleich zur Trepanation schreiten will.

\section{Nachtrag bei der Korrektur.}

Den hier geschilderten Erfahrungen möchte ich eine neue Beobachtung aus letzter Zeit anfügen, welche die differentiell-diagnostische Schwierigkeit zwischen Neubildung der hinteren Schädelgrube speziell Kleinhirntumor und idiopathischem Hydrocephalus weiterhin bestätigt.

Ein 24 jähriger Lehrer klagt, ohne dass Vorboten bestanden hatten, in den ersten Junitagen über Unwohlsein, Uebelkeit. Tags darauf treten Kopfschmerzen und Erbrechen auf. Er fühlt sich teilnahmlos und unlustig und hatte von Anfang an das Gefühl eines unsicheren Ganges. Die Beschwerden waren zunächst erträglich, so dass er seinen Dienst wieder aufnahm, doch fiel ihm dabei auf, dass er nicht stehen konnte, ohne sich anzulehnen. Er tat aber noch Dienst bis zum 21., dann suohte er das Krankenhaus auf. Es fiel ihm anf, dass die Sprache schlechter wurde und dass die Feder beim Schreiben ausfahrende Bewegungen machte. Auch der Mutter fiel das langsame Sprechen auf. Die Kopfschmerzen bestanden fort und sassen anfangs in der Stirn, später im Hinterkopf und der rechten Schläfe. Temperatur'steigerungen hatten nicht bestanden. Die starke Zunahme der Kopfschmerzen veranlasste ihn, die Klinik aufzusuchen.

Hier wurde er am 27. Juni aufgenommen und machte noch folgende Angaben: Er sei abgesehen von einer 4 wöchigen fieberhaften Lungenerkrankung vor 7 Jahren immer gesund gewesen. Bezüglich der jetzt vorliegenden Erkrankung gab er an, dass die Augen in den letzten Tagen schlechter geworden seien, er sehe weniger deutlich wie durch einen Schleier. Kopfschmerzen und Erbrechen seien fast unerträglich geworden.

Die Sprache ist langsam, gelegentlich skandierend.

Die objektive Untersuchung ergibt an den inneren Organen normalen Befund. Puls 52, voll, ziemlich gespannt. 
Der Kopf ist nicht klopfempfindlich, Trigeminus- und Occipitalisaustrittsstellen beiderseits druckempfindlich. Der Kopf wird steif gehalten. Beschränkung der Kopfbewegungen in sämtlichen Dimensionen. Nackenmuskulatur nicht druclempfindïich. Zunehmende Kopfschmerzen bei passiven Bewegungen. Auch bei dem Aufrichten aus dem Liegen zunehmende Schmerzen.

Die Augenbewegungen sind frei, kein Nystagmus, Konvergenzbewegung schlecht. Keine Doppelbilder. Die Pupillen mittelweit und von guter Lichtreaktion. Am Augenhintergrund am ersten Tage kein sicher pathologischer Befund, am zweiten unscharfe Begrenzung temporalwärts und verstärkte Gefässfüllung.

Kornealreflex ist rechts lebhaft, links sehr schwach, links wird die Berührung der Kornea weniger dentlich empfunden. Für feine Berührung scheint im Gebiet des II. Quintusastes Herabsetzung zu bestehen. Motorischer Quintus gut. Masseterreflex lebhaft. Der linke Mundfazialis ist schlechter innerviert, als der rechte. Die Zunge ist belegt, weicht nicht ab.

Grobe Kraft an Armen ond Beinen sehr gut. Etwas Ataxie an den Beinen und beim Nasenfingerversuch beiderseits, aber links deutlicher. Keine Störung der Bewegungsempfindung. Taumelnder Gang ohne Vorliebe für eine Richtung. Pat. kann nicht ohne Unterstätzung stehen. Taumeln wird bei Augenschluss nicht stärker.

Patellar- und Achillesreflexe beiderseits gleich, Babinski unsicher, mitunter links angedeutet.

Häufiges Erbrechen, anfallsweise Steigerung der Kopfschmerzen.

ln den folgenden Tagen zunehmende Kopfschmerzen. Beim Aufheben lautes Jammern, erbricht viel. Zeitweise ganz unregelmässige Atmung. Pat. bittet um Operation wegen der Schmerzen.

Psychisch ist Pat. zeitweise euphorisch, er wird mehr und mehr schlafsüchtig und schwer erweckbar.

Somatischer Befund im wesentlichen derselbe. Die Augenhintergrundveränderungen nehmen zu. Die Begrenzung der Papille wird noch unscharfer. Der Gang wird noch schlechter.

Babinskischer Reflex links deutlicher. Lokale Druckempfindlichkeit des 4.-7. Proc. spin. dorsal.

Lumbalpunktat: Liquor klar. Keine Druckvermehrung. Fuchs-Rosenthal 5 Lymphozyten, im Nisslpräparat keine Vermehrung. Keine Eiweissvermehrung. Serologisch: Blut und Liquor negativ.

Pat. wird wegen der Zunahme der Kopfsschmerzen, des Erbrechens, beginnender Somnolenz und der Staunngspapille zur Entlastungsoperation in die chirurgische Klinik verlegt. Es wird an Kleinhirntumor gedacht. Dort Eröffnung über dem Kleinhirn. Starke Vorwölbung. der Dura. Kleinhirn quillt nach Eröffnung der Dura sehr stark vor. Ein Tumor ist nioht zu sehen. Schluss des Hautlappens.

Exitus etwa 24 Stunden nach der Operation im Koma.

Obdulition: Hydrocephalus idiopath. mittleren Grades, vor allem des III. und der Seitenventrikel, sonst liein abnormer Befund am Gehirn. 
Der Fall bestätigt wieder die Erfahrung, dass es klinisch nicht mit Sicherheit möglich ist, den idiopathischen Hydrocephalus von Tumoren der hinteren Schädelgrube zu unterscheiden. Vorangegangene Traumen, akute Infektionen, überhaupt jeder ätiologische Hinweis fehlten. Gewiss konnte die scbnelle Entwicklung verdächtig erscheinen. Aber wie häufig ist auch beim Tumor eine rapide Entwicklung der Hirnsymptome. Man sieht das besonders dann, wenn Hydrocephalusentwicklung hinzutriti, wenn es sich um cystöse Bildungen oder um Blutungen in den Tumor handelt. Gerade von den besprochenen Neubildungen im IV. Ventrikel ist behauptet worden, dass sie zunächst symptomlos bleiben und dann eine akuteste Steigerung zeigen können. Auch auf die Druckempfindlichkeit der Processus spinosi, die am 3. Tage nach der Aufnahme beobachtet wurden, soll im Hinblick auf die Bedeutung, die ihr Oppenheim für die Hydrocephalusdiagnose zuspricht, hingewiesen werden. Entscheidende Bedeutung wird man auch diesem Symptom nicht beilegen, da es wohl kaum anders denn als Ausdruck der Liquorspannung aufzufassen ist. Auch der Liquorbefund selbst batte nichts Eindentiges. Immerhin kann man wohl sagen, dass das Fehlen der Globulinreaktion und der Eiweissvermehrung vielleicht bei Tumor ungewöhnlicher ist, als beim idiopathischen Hydrocephalus. Aber schon unsere wenigen Fälle zeigen, dass ein sicheres Kriterium auch hierin nicht liegt.

Die Lokalsymptome sprachen deutlich für die hintere Schädelgrube speziell für das Kleinhirn. Von Anfang an, schon ebe Benommenheit bestand, stand die statische Ataxie im Vordergrund; die Ataxie der Extremitäten ohne Lageempfindungsstörungen, die Herabsetzung des Kornealreflexes, die Zunahme der Kopfschmerzen bei Lokomotionen, insbesondere beim Sicherheben waren in diesem Sinne zu deuten. Auffallend war das Fehlen des Nystagmus. Auch hier fand sich wieder die Halbseitigkeit einzelner Symptome, ohne dass der Befund bei der Obduktion etwa in der stärkeren Ausbildung des Hydrocephalus auf einer Seite eine Erklärung gab.

Die praktische Bedeutung der Unsicherheit dieser Differentialdiagnose zu illustrieren, ist dieser Fall besonders geeignet. Denn wäre die Diagnose Hydrocephalus idiopathicus sicher zu stellen gewesen, so hätte ich zunächst doch noch den Versuch einer energischen internen Therapie gemacht und mich im Falle der Ergebnislosigkeit einer solchen wahrscheinlich mit der Ventrikeldrainage begnügt. Freilich wäre man auch dann vor der Ueberraschung des plötzlichen Todes nicht sicher gewesen. Aber nach dem Stande unserer Kenntnisse wird man in so bedrohlich aussehenden Fällen sich an die grössere Wahrscheinlichkeit zu 
halten und seine Indikationen darnach zu gestaltej haben. Die grössere Wahrscheinlichkeit hatte aber der Tumor und da war bei der gefahrdrohenden Symptomenentwicklung das rasche chirurgische Eingehen indiziert.

\section{Literaturverzeichnis.}

Anton, Dieses Archiv. 48. Bd.

A. Stern, Ueber Tumoren des IV. Ventrikels. Deutsche Zeitschr. tür Nervenheilkunde. 34. Bd.

A. Stern, Deutsche Zeitschr. f. klin. Med. 1907.

Boström, Zentralbl. f. allgem. Pathol. und pathol. Anat. 8. Bd.

Brüning, Zur Kasuistik der Tumoren im IV. Ventrikel. Jahrbuch f. Kinderheilkunde. 1902.

Cimbal, Zur Lehre von den Geschwülsten des IV. Ventrikels. Inang.-Dissert. Breslau. 1901.

Henneberg, Ueber den Rautengrubencysticercus. Monatsschr. f. Psychiatrie und Neurol. 1906.

Henneberg, Ventrikel- und Ponstumoren. Charité-Annalen. 27. Bd.

Frick, Ein Fall von Cholesteatom im IV. Ventrikel. Inaug.-Dissert. Breslau. 1910.

Finkelnburg, Deutsche Zeitschr. f. Nervenheilkunde. 21. Bd.

Hensen, Ueber Zystizerken im 4. Ventrikel. Deutsches Arch. f. klin. Med.

Oppenheim, Lebrbuch für Nervenkrankheiten. 1909.

Oppenheim, Monatsschr. f. Psych. und Neurol. 1906.

Völsch, Neurol. Zentralbl. 1909.

Contella, Tumeur du quatrième ventricule. Arch. d'ophthalm. 1909.

Uhthoff, Gräfe-Sämisch Handbuch. XI. Bd. II.

Bruns, Die Geschwülste des Nervensystems. 1908. 